\title{
Ten years of research in spectrum sensing and sharing in cognitive radio
}

\author{
Lu Lu*, Xiangwei Zhou, Uzoma Onunkwo and Geoffrey Ye Li
}

\begin{abstract}
Cognitive radio (CR) can successfully deal with the growing demand and scarcity of the wireless spectrum. To exploit limited spectrum efficiently, CR technology allows unlicensed users to access licensed spectrum bands. Since licensed users have priorities to use the bands, the unlicensed users need to continuously monitor the licensed users' activities to avoid interference and collisions. How to obtain reliable results of the licensed users' activities is the main task for spectrum sensing. Based on the sensing results, the unlicensed users should adapt their transmit powers and access strategies to protect the licensed communications. The requirement naturally presents challenges to the implementation of CR. In this article, we provide an overview of recent research achievements of including spectrum sensing, sharing techniques and the applications of CR systems.
\end{abstract}

Keywords: cognitive radio, cooperative communications, spectrum sensing, spectrum sharing.

\section{Introduction}

Due to the rapid growth of wireless communications, more and more spectrum resources are needed. Within the current spectrum framework, most of the spectrum bands are exclusively allocated to specific licensed services. However, a lot of licensed bands, such as those for TV broadcasting, are underutilized, resulting in spectrum wastage [1]. This has promoted Federal Communications Commission (FCC) to open the licensed bands to unlicensed users through the use of cognitive radio (CR) technology [2-6]. The IEEE 802.22 working group [7] has been formed to develop the air interference for opportunistic secondary access to TV bands.

In practice, the unlicensed users, also called secondary users (SUs), need to continuously monitor the activities of the licensed users, also called primary users (PUs), to find the spectrum holes ( $\mathrm{SHs}$ ), which is defined as the spectrum bands that can be used by the SUs without interfering with the PUs. This procedure is called spectrum sensing [8-10]. There are two types of SHs, namely temporal and spatial SHs [9], respectively. A temporal $\mathrm{SH}$ appears when there is no $\mathrm{PU}$ transmission during a certain time period and the SUs can use the spectrum for transmission. A spatial $\mathrm{SH}$ appears when the $\mathrm{PU}$

\footnotetext{
* Correspondence: lulu0528@gatech.edu

School of Electrical and Computer Engineering, Georgia Institute of Technology, Atlanta, GA 30332-0250, USA
}

transmission is within an area and the SUs can use the spectrum outside that area.

To determine the presence or absence of the PU transmission, different spectrum sensing techniques have been used, such as matched filtering detection, energy detection, and feature detection [11]. However, the performance of spectrum sensing is limited by noise uncertainty, multipath fading, and shadowing, which are the fundamental characteristics of wireless channels. To address this problem, cooperative spectrum sensing (CSS) has been proposed [12] by allowing the collaboration of SUs to make decisions.

Based on the sensing results, SUs can obtain information about the channels that they can access. However, the channel conditions may change rapidly and the behavior of the PUs might change as well. To use the spectrum bands effectively after they are found available, spectrum sharing and allocation techniques are important $[6,13]$. As PUs have priorities to use the spectrum when SUs co-exist with them, the interference generated by the SU transmission needs to be below a tolerable threshold of the PU system [14]. Thus, to manage the interference to the PU system and the mutual interference among SUs, power control schemes should be carefully designed. By utilizing advanced technologies such as multiple-input multiple-output (MIMO) and beamforming with smart antenna, interference-free 
co-exiting transmission can be achieved [15]. In the multi-hop CR system, relays can assist SUs' transmission, which generate spatial SHs and help to achieve more communication opportunities. Moreover, the resource competition among SUs needs to be addressed.

There are a lot of progresses on CR technology in the last ten years. This article provides an overview of some recent techniques, potential challenges, and future applications of CR. In Section 2, fundamental spectrum sensing techniques are provided. In Section 3, CSS techniques to boost the sensing performance are presented. Spectrum sharing and allocation schemes are discussed in Section 4. The applications of CR technology and conclusions are in Sections 5 and 6, respectively.

Table 1 lists some abbreviations that have been or will be used in this article.

\section{Local spectrum sensing}

Spectrum sensing enables SUs to identify the SHs, which is a critical element in CR design $[9,10,16]$. Figure 1 shows the principle of spectrum sensing. In the figure, the PU transmitter is sending data to the PU receiver in a licensed spectrum band while a pair of SUs intends to access the spectrum. To protect the PU transmission, the SU transmitter needs to perform spectrum sensing to detect whether there is a PU receiver in the coverage of the SU transmitter.

Instead of detecting PU receiver directly, the SU transmitter can detect the presence or absence of PU signals easily. However, as shown in Figure 1, the radius of PU transmitter and PU receiver detections are different, which lead to some shortcomings and challenges. It may happen that the PU receiver is outside the PU transmitter detection radius, where the SH may be missed. Since the PU receiver detection is difficult, most study focuses on PU transmitter detection $[6,13]$.

It is worth noting that, in general, it is difficult for the SUs to differentiate the PU signals from other pre-existing SU transmitter signals. Therefore, we treat them all as one received signal, $s(t)$. The received signal at the $\mathrm{SU}, x(t)$, can be expressed as [17]

$$
x(t)=\left\{\begin{array}{cc}
n(t) & \mathcal{H}_{0} \\
s(t)+n(t) & \mathcal{H}_{1}
\end{array}\right.
$$

where $n(t)$ is the additive white Gaussian noise (AWGN). $\mathcal{H}_{0}$ and $\mathcal{H}_{1}$ denote the hypotheses of the absence and presence of the PU signals, respectively. The objective for spectrum sensing is to decide between $\mathcal{H}_{0}$ and $\mathcal{H}_{1}$ based on the observation $x(t)$.
Table 1 List of abbreviations

\begin{tabular}{|c|c|}
\hline $\mathrm{AF}$ & Amplify-and-forward \\
\hline AWGN & Additive white Gaussian noise \\
\hline BS & Base station \\
\hline CAF & Cyclic autocorrelation function \\
\hline$C R$ & Cognitive radio \\
\hline CSD & Cyclic spectrum density \\
\hline CSS & Cooperative spectrum sensing \\
\hline DoD & Department of defense \\
\hline DR & Detect-and-relay \\
\hline ECC & Electronic Communications Committee \\
\hline FCC & Federal Communications Commission \\
\hline GLRT & Generalized likelihood ratio test \\
\hline HDD & Hard-decision-based detection \\
\hline IPC & Interference powers constraint \\
\hline LRT & Likelihood ratio test \\
\hline LTE-A & LTE-Advanced \\
\hline MIMO & Multiple-input multiple-output \\
\hline MLE & Maximum likelihood estimate \\
\hline MME & Maximum-minimum eigenvalue \\
\hline MMSE & Minimum mean-square-error \\
\hline $\mathrm{NE}$ & Nash equilibrium \\
\hline NP & Neyman-Pearson \\
\hline Ofcom & Office of Communications \\
\hline OFDMA & Orthogonal frequency division multiple access \\
\hline OPC & Outage probability constraint \\
\hline OSA & Opportunistic spectrum access \\
\hline OSO & Opportunistic spatial orthogonalization \\
\hline PDF & Probability density function \\
\hline PSD & Power spectral density \\
\hline PU & Primary user \\
\hline QAM & Quadrature amplitude modulation \\
\hline Qos & Quality of service \\
\hline QCQP & Quadratically constrained quadratic problem \\
\hline RF & Radio-front \\
\hline RLC & Rate loss constraint \\
\hline $\mathrm{ROC}$ & Receiver operating characteristics \\
\hline SBS & Secondary base station \\
\hline SDD & Soft-decision-based detection \\
\hline SDR & Semidefinite relaxation \\
\hline SFD & Spectral feature detector \\
\hline $\mathrm{SH}$ & Spectrum holes \\
\hline SINR & Signal-to-interference-plus-noise ratio \\
\hline SNR & Signal-to-noise ratio \\
\hline SPRT & Sequential probability ratio test \\
\hline SU & Secondary user \\
\hline TPA & Transmit power allocation \\
\hline TPC & Transmit power constraint \\
\hline$X G$ & Next generation \\
\hline
\end{tabular}




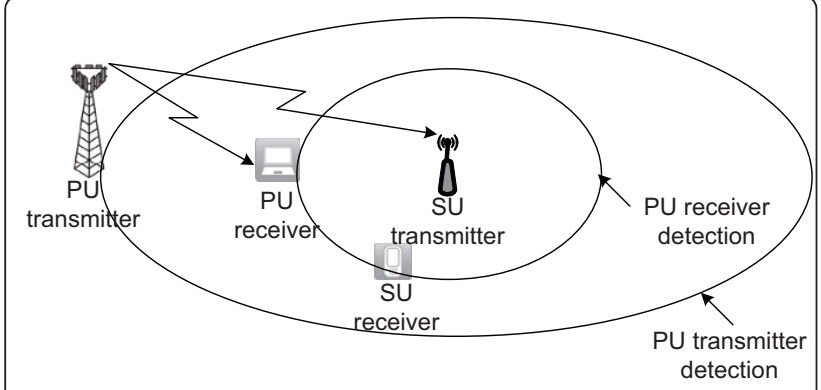

Figure 1 Spectrum sensing concept.

The detection performance is characterized by the probabilities of detection, $P_{d}$, and false-alarm, $P_{f} . P_{d}$ is the probability that the decision is $\mathcal{H}_{1}$, while $\mathcal{H}_{1}$ is true; $P_{f}$ denotes the probability that the decision is $\mathcal{H}_{1}$, while $\mathcal{H}_{0}$ is true. Based on $P_{d}$, the probability of missdetection $P_{m}$ can be obtained by $P_{m}=1-P_{d}$.

\subsection{Hypothesis testing criteria}

There are two basic hypothesis testing criteria in spectrum sensing: the Neyman-Pearson (NP) and Bayes tests. The NP test aims at maximizing $P_{d}$ (or minimizing $\left.P_{m}\right)$ under the constraint of $P_{f} \leq \alpha$, where $\alpha$ is the maximum false alarm probability. The Bayes test minimizes the average cost given by $\mathcal{R}=\sum_{i=0}^{1} \sum_{j=0}^{1} C_{i j} \operatorname{Pr}\left(\mathcal{H}_{i} \mid \mathcal{H}_{j}\right) \operatorname{Pr}\left(\mathcal{H}_{j}\right)$, where $C_{i j}$ are the cost of declaring $\mathcal{H}_{i}$ when $\mathcal{H}_{j}$ is true, $\operatorname{Pr}\left(\mathcal{H}_{i}\right)$ is the prior probability of hypothesis $\mathcal{H}_{i}$ and $\operatorname{Pr}\left(\mathcal{H}_{i} \mid \mathcal{H}_{j}\right)$ is the probability of declaring $\mathcal{H}_{i}$ when $\mathcal{H}_{j}$ is true. Both of them are equivalent to the likelihood ratio test (LRT) [18] given by

$$
\Lambda(\mathbf{x})=\frac{P\left(\mathbf{x} \mid \mathcal{H}_{1}\right)}{P\left(\mathbf{x} \mid \mathcal{H}_{0}\right)}=\frac{P\left(x(1), x(2), \ldots, x(M) \mid \mathcal{H}_{1}\right)}{P\left(x(1), x(2), \ldots, x(M) \mid \mathcal{H}_{0}\right)} \underset{\mathcal{H}_{0}}{\stackrel{\mathcal{H}_{1}}{\gtrless}} \chi(2)
$$

where $P\left(x(1), x(2), \ldots, x(M) \mid \mathcal{H}_{i}\right)$ is the distribution of observations $\mathrm{x}=[x(1), x(2), \ldots, x(M)]^{T}$ under hypothesis $\mathcal{H}_{i}, i \in\{0,1\}, \Lambda(\mathbf{x})$ is the likelihood ratio, $M$ is the number of samples, and $\gamma$ is the detection threshold, which is determined by the maximum false alarm probability, $\alpha$, in NP test and $\gamma=\frac{\operatorname{Pr}\left(\mathcal{H}_{0}\right)\left(C_{10}-C_{00}\right)}{\operatorname{Pr}\left(\mathcal{H}_{1}\right)\left(C_{01}-C_{11}\right)}$ in the Bayes test.

In both tests, the distributions of $P\left(\mathbf{x} \mid \mathcal{H}_{i}\right), i \in\{0,1\}$, are known. When there are unknown parameters in the probability density functions (PDFs), the test is called composite hypothesis testing. Generalized likelihood ratio test (GLRT) is one kind of the composite hypothesis test. In the GLRT, the unknown parameters are determined by the maximum likelihood estimates (MLE) [19-21]. GLRT detectors have been proposed for multi- antenna systems in [19] and for sensing OFDM signals in $[20,21]$ by taking some of the system parameters, such as channel gains, noise variance, and PU signal variance as the unknown parameters.

Sequential testing is another type of hypothesis testing, which requires a variable number of samples to make decisions. The sequential probability ratio test (SPRT) minimizes the sensing time subject to the detection performance constraints [22]. In the SPRT, samples are taken sequentially and the test statistics are compared with two threshold $\gamma_{0}$ and $\gamma_{1}\left(\gamma_{0}<\gamma_{1}\right)$, which are determined by the detection requirements. Using the SPRT, the SU makes decisions according to the following rule: $\mathcal{H}_{1}$ if $\Lambda(\mathbf{x})>\gamma_{1} ; \mathcal{H}_{0}$ if $\Lambda(\mathbf{x})<\gamma_{0}$; more samples are needed if $\gamma_{0}<\Lambda(\mathbf{x})<\gamma_{1}$. General sequence detection algorithms for Markov sources with noise have been proposed in [23]. A weighted, soft-input sequence detection algorithm based on forward-backward procedure is shown to be optimal in minimizing the Bayesian risk when different Bayesian cost factors are assigned for missed detection and false alarm. Moreover, a new limitation, called risk floor, has been discovered for traditional physical layer sensing schemes, which is caused by finite channel dwell time, where longer observation windows are more likely to mix the PU's behavior from multiple states, leading to degraded performance.

\subsection{Local spectrum sensing techniques}

To identify the SHs and protect PU transmission, different local spectrum sensing techniques have been proposed for individual SUs by applying the hypothesis testing criteria discussed above.

\subsubsection{Matched filtering detector}

If the SUs know information about the PU signal, the optimal detection method is matched filtering [11], which correlates the known primary signal with the received signal to detect the presence of the PU signal and thus maximize the signal-to-noise ratio (SNR). The matched filtering detector requires short sensing time to achieve good detection performance. However, it needs knowledge of the transmit signal by PU that may not be known at the SUs. Thus, the matched filtering technique is not applicable when transmit signals by the PUS are unknown to the SUs.

\subsubsection{Energy detector}

Energy detector [11] is the most common spectrum sensing method. The decision statistics of the energy detector are defined as the average energy of the observed samples

$$
Y=\frac{1}{N} \sum_{t=1}^{N}|x(t)|^{2}
$$


The decision is made by comparing $Y$ with a threshold, $\gamma$. If $Y \geq \gamma$, the SU makes a decision that the PU signal is present $\left(\mathcal{H}_{1}\right)$; otherwise, it declares that the PU signal is absent $\left(\mathcal{H}_{0}\right)$.

The energy detector is easy to implement and requires no prior information about the PU signal. However, the uncertainty of noise power imposes fundamental limitations on the performance of the energy detector [24-26]. Below an SNR threshold, a reliable detection cannot be achieved by increasing the sensing duration. This SNR threshold for the detector is called SNR wall [24]. With the help of the PU signal information, the SNR wall can be mitigated, but it cannot be eliminated [25]. Moreover, the energy detector cannot distinguish the PU signal from the noise and other interference signals, which may lead to a high false-alarm probability.

\subsubsection{Feature detector}

Cyclostationary detector is one of the feature detectors that utilize the cyclostationary feature of the signals for spectrum sensing $[27,28]$. It can be realized by analyzing the cyclic autocorrelation function (CAF) of the received signal $x(t)$, expressed as

$$
R_{x}^{(\beta)}(\tau)=E\left[x(t) x^{*}(t-\tau) e^{-j 2 \pi \beta t}\right],
$$

where $E[\cdot]$ is the expectation operation, * denotes complex conjugation, and $\beta$ is the cyclic frequency. CAF can also be represented by its Fourier series expansion, called cyclic spectrum density (CSD) function [29], denoted as

$$
S(f, \beta)=\sum_{\tau=-\infty}^{+\infty} R_{x}^{(\beta)}(\tau) e^{-j 2 \pi f \tau} .
$$

The CSD function exhibits peaks when the cyclic frequency, $\beta$, equals the fundamental frequencies of the transmitted signal. Under hypothesis $\mathcal{H}_{0}$, the CSD function does not have any peaks since the noise is, in general, non-cyclostationary.

Generally, feature detector can distinguish noise from the PU signals and can be used for detecting weak signals at a very low SNR region, where the energy detection and matched filtering detection are not applicable. In [30], a spectral feature detector (SFD) has been proposed to detect low SNR television broadcasting signals. The basic strategy of the SFD is to correlate the periodogram of the received signal with the selected spectral features of a particular transmission scheme. The proposed SFD is asymptotically optimal according to the NP test, but with lower computational complexity.

To capture the advantages of the energy detector and the cyclostationary detector while avoiding the disadvantages of them, a hybrid architecture, associating both of them, for spectrum sensing has been proposed in [31].
It consists of two stages: an energy detection stage that reflects the uncertainty of the noise and a cyclostationary detection stage that works when the energy detection fails. The proposed hybrid architecture can detect the signal efficiently.

\subsubsection{Other techniques}

There are several other spectrum sensing techniques, such as eigenvalue-based and moment-based detectors.

In a multiple-antenna system, eigenvalue-based detection can be used for spectrum sensing [32,33]. In [32], maximum-minimum eigenvalue and energy with minimum eigenvalue detectors have been proposed, which can simultaneously achieve both high probability of detection and low probability of false-alarm without requiring information of the PU signals and noise power. In most of the existing eigenvalue-based methods, the expression for the decision threshold and the probabilities of detection and false-alarm are calculated based on the asymptotical distributions of eigenvalues. To address this issue, the exact decision threshold for the probability of false-alarm for the MME detector with finite numbers of cooperative SUs and samples has been derived in [33], which will be discussed in Section 3.

When accurate noise variance and PU signal power are unknown, blind moment-based spectrum sensing algorithms can be applied [34]. Unknown parameters are first estimated by exploiting the constellation of the PU signal. When the SU does not know the PU signal constellation, a robust approach that approximates a finite quadrature amplitude modulation (QAM) constellation by a continuous uniform distribution has been developed [34].

\subsection{Sensing scheduling}

When and how to sense the channel are also crucial for spectrum sensing. Usually, short quiet periods are arranged inside frames to perform a coarse intra-frame sensing as a pre-stage for fine inter-frame sensing [35]. Accordingly, intra-frame sensing is performed when the SU system is quiet and its performance depends on the sample size in the quiet periods. The frame structure for CR network is shown in Figure 2. Based on this structure, there are sensing-transmission tradeoff problems. Under the constraint of PU system protection, the optimal sensing time to maximize the throughput [36] and to minimize outage probability [37] of the SU system have been studied, respectively.

However, there are some problems about the conventional structure: (1) the sample size of the quiet periods may not be enough to get good sensing performance; (2) all CR communications have to be postponed during channel sensing; (3) the placement of the quiet periods causes an additional burden of synchronization. To 


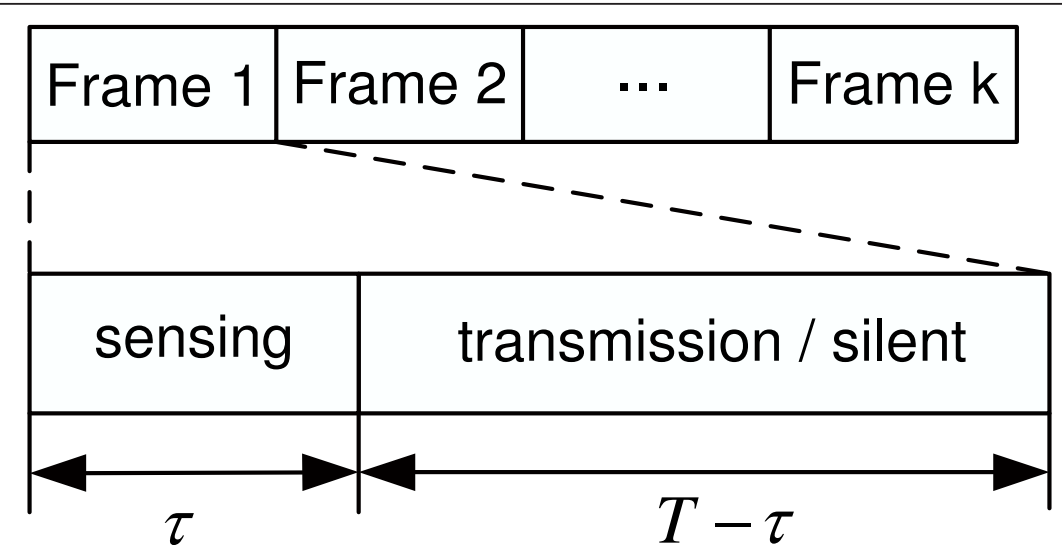

Figure 2 Frame structure for periodic spectrum sensing, where $\tau$ denotes the sensing period and $T-\tau$ denotes the data transmission or silent period.

address these problems, novel spectrum sensing scheduling schemes have been proposed.

In [38], adaptively scheduling spectrum sensing and transmitting data schemes have been proposed to minimize the negative effect caused by the traditional structure. The spectrum sensing is carried out when the channels are in poor conditions and data are transmitted when the channels are good. In [39], sensing period has been optimized to make full use of opportunities in the licensed bands. Moreover, a channel-sequencing algorithm has been proposed to reduce the delay in searching for an idle channel. To increase the sample size, quiet-active and active sensing schemes have been proposed [40]. In the quiet-active sensing scheme, the inactive SUs sense the channel in both the quiet and active data transmission periods. To fully avoid synchronization of quiet-periods, pure active sensing has been proposed where the quiet periods are replaced by "quiet samples" in other domains, such as quiet sub-carriers in orthogonal frequency division multiple access (OFDMA) systems.

\subsection{Challenges}

\subsubsection{Wideband sensing}

Wideband sensing faces technical challenges and there is limited work on it. The main challenge stems from the high data rate radio-front (RF) end requirement to sense the whole band, with the additional constraint that deployed CR systems (like mobile phones) will be limited in data processing rates. To achieve reliable results, the sample rate should be above the Nyquist rate if conventional estimation methods are used, which is a challenging task. Alternatively, the RF end can use a sequence of narrowband bandpass filters to turn a wideband signal into narrow-band ones and sense each of them [41]. However, a large number of RF components are needed for the whole band. For more effective SU networks, a multiband sensing-time-adaptive joint detection framework has been proposed in $[42,43]$, which adaptively senses multiple narrowband channels jointly to maximize the achievable opportunistic throughput of the SU network while keeping the interference with the PU network bounded to a reasonably low level. Based on energy detector for narrowband sensing, the sensing time and detection thresholds for each narrowband detector are optimized jointly, which is different from the previous multiband joint detection framework in [44].

\subsubsection{Synchronization}

Besides the synchronization issue for quiet sensing period, spectrum synchronization before the data transmission for non-contiguous OFDM based systems is also a challenge. To address this challenge [45], received training symbols can be used to calculate a posterior probability of each subband's being active without the information of out-of-band spectrum synchronization. The proposed hard-decision-based detection (HDD) utilizes a set of adjacent subbands while the soft-decisionbased detection (SDD) uses all the subbands for detection. Both HDD and SDD schemes provide satisfactory performance while the SDD performs better.

\section{Cooperative spectrum sensing}

The performance of spectrum sensing is limited by noise uncertainty, multipath fading, and shadowing, which are the fundamental characteristics of wireless channels. If the PU signal experiences deep fading or blocked by obstacles, the power of the received PU signal at the SU may be too weak to be detected, such as the case for $\mathrm{SU}_{3}$ as shown in Figure 3. If the $\mathrm{SU}$ 


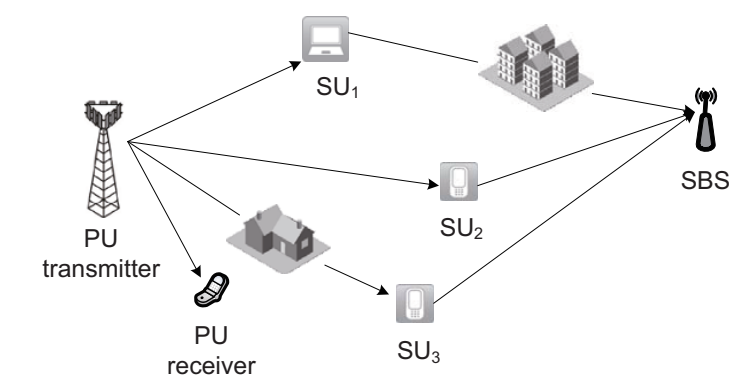

Figure 3 Cooperative spectrum sensing model, where $\mathrm{SU}_{1}$ is shadowed over the reporting channel to the secondary base station (SBS) and $\mathrm{SU}_{3}$ is shadowed over the sensing channel.

transmitter cannot detect the presence of the PU transmitter while the PU receiver is within the transmission range of the $\mathrm{SU}$, the transmission of the $\mathrm{PU}$ will be interfered. To address this problem, CSS has been proposed [12]. With the collaboration of several SUs for spectrum sensing, the detection performance will be improved by taking advantage of independent fading channels and multiuser diversity. Based on the decision fusion criteria, CSS can be realized in either a centralized or a distributed manner.

\subsection{Centralized CSS}

A centralized CSS system consists of a secondary base station (SBS) and a number of SUs. In this system, the SUs first send back the sensing information to the SBS. After that, the SBS will make a decision on the presence or absence of the PU signal based on its received information and informs the SUs about the decision.

\subsubsection{Data fusion schemes}

Different data fusion schemes for CSS have been studied. Reporting data from the SUs may be of different forms, types, and sizes. In general, the sensing information combination at the SBS can be categorized as soft combination and hard combination techniques.

Soft Combination In soft combination, the SUs can send their original or processed sensing data to the SBS [4]. To reduce the feedback overhead and computational complexity, various soft combination schemes based on energy detection have been investigated [46]. In these schemes, each SU sends its quantized observed energy of the received signal to the SBS. By utilizing LRT at the SBS, the obtained optimal soft combination decision is based on a weighted summation of those energies.

At the SBS, linear combination of the test statistics from the SUs is the most common fusion rule. The global test statistic of linear combination is

$$
Y_{c}=\sum_{j=1}^{N} w_{j} Y_{j}
$$

where $Y_{j}$ is the local test statistics (e.g. received energy in energy detection or in matched filtering $[47,48]$ ) from $\mathrm{SU}_{j}$, and $w_{j}$ is the weight. $Y_{c}$ is compared with a threshold, $\gamma_{c}$, to make decisions. The optimization of linear CSS for the general model is non-convex and challenging. According to a taxonomy based on the probabilities of false alarm and detection, three kinds of CR systems have been developed, namely conservative, aggressive, and hostile [47]. Since the last kind is too complex and of limited interest in applications, only the first two have been studied in [47]. Recently, a general model for all the modes has been investigated in [48]. The problem of determining the weights to maximize the detection probability under a given targeted falsealarm probability has been studied. Based on the solution of a polynomial equation, the global optimum is found by an explicit algorithm.

The linear CSS design does not only focus on the detection probability optimization but also the tradeoff in sensing time setting. In a multi-channel system based on linear CSS, the optimal value of the decision threshold, $\gamma_{c}$, and sensing time, $\tau$, are obtained by maximizing the throughput of the SU system for a given detection probability [49]. The original non-convex problems can be successfully converted into convex subproblems. To avoid the convex approximation, an alternative optimization technique based on genetic algorithms [50] has been proposed to directly search for the optimal solution.

Although soft combination schemes can provide good detection performance, the overhead for feedback information is high. It makes the CSS impractical under a large number of cooperative SUs. A soften-hard combination with two-bit overhead [46] has been proposed to provide comparable performance with less complexity and overhead.

Hard combination For hard combination, the SUs feed back their own binary decision results to the SBS. Let $u_{i}$ denotes the local decision of $\mathrm{SU}_{i}$, where $u_{i}=1$ and 0 indicate the presence $\left(\mathcal{H}_{1}\right)$ and the absence $\left(\mathcal{H}_{0}\right)$ of the PU signal, respectively. $u$ denotes the decision of the SBS. The most common fusion rules are OR-rule, ANDrule, and majority rule. Under the OR-rule, $u=1$ if there exists $u_{i}=1$. The AND-rule refers to the SBS determines $u=1$ if $u_{i}=1$, for all $i$. For the majority rule, if more than half of the SUs report $u_{i}=1$, the SBS decides $u=1$. These fusion rules can be generalized to a $K$-out-of- $N$ rule, where $u=1$ if $K$ out of $N$ SUs report the presence of the PU signal. When $K=1$ and $K=N$, the $K$-out-of- $N$ rule becomes the OR-rule and the AND-rule, respectively.

When the OR-rule or the AND-rule is used, the threshold of detector should be adjusted according to $N$ 
to get better performance than a non-cooperative system [51]. For the $K$-out-of- $N$ rule, the optimal value of $K$ and sensing time are obtained in [52] by maximizing the average achievable throughput of the SU system subject to a detection performance requirement. When all the SUs employ identical constant detection threshold, an optimal $K$ has been derived to improve both false-alarm and miss-detection probabilities in [53].

When the SUs have different detection SNRs, it is not efficient to use the $K$-out-of- $N$ fusion rule since it ignores the difference between decisions from a SU with high detection SNR and a SU with low detection SNR. Weighted decision fusion schemes have been proposed to take into account the difference in the reliability of the decisions made by different SUs [54], which are reflected in the weights of the decisions at the SBS. The optimal fusion rules in three different scenarios have been derived during the optimization of the sensingthroughput tradeoff problem. To ensure reliable detection, the correlation among different SUs should also be taken into consideration. A linear-quadratic fusion strategy has been proposed in [55] to exploit the correlation, which significantly enhances the detection performance.

\subsubsection{User selection}

User selection in CSS is crucial. Since SUs are located differently and strengths of received PU signals are different, it is shown in [51] that cooperation of all the SUs is not optimal. The optimal detection/false-alarm probabilities are achieved by selectively cooperating among SUs with high detection SNRs of the PU signal. The user selection is hard for the detection of smallscale PU signals that have small-footprint due to their weak power and unpredictability of spatial and temporal spectrum usage patterns [56]. Data-fusion range is identified as a key factor that enables effective CSS. The SUs in the data-fusion range cooperate to sense PU signals while others do not [56].

In multi-channel CR networks, it is impractical to make SUs to sense all the channels. The multi-channel coordination issues, such as, how to assign SUs to sense channels and to maximize the expected transmission time, have been studied in [57]. It has been shown that multi-channel coordination can improve CSS performance. Similar issues can be also found in sensor networks [58].

If the SUs cannot distinguish the signals from the PU and other SUs, it may lose the opportunity to access the spectrum [59]. The presence/absence of possible interference from other SU transmitters is a major component of the uncertainty limiting the detection performance. Coordinating the nearby SUs can reduce the uncertainty [59].

\subsubsection{Sequential CSS}

In CSS, SPRT can opportunistically reduce the sample size required to meet the reliability target. In [60], sequential detection scheme has been designed to minimize the detection time. In the scheme, each SU calculates the log-likelihood ratio of its measurement and the SBS accumulates these statistics to determine whether or not to stop making measurements. A robust design is developed for the scenarios with unknown system parameters, such as noise variance and signal power. Moreover, a tradeoff between sensing time and average data rate of the SUs based on sequential sensing for multichannel system has been studied in [61]. A stopping policy and an access policy are given to maximize the total achievable rate of the SU system under a misdetection probability constraint for each channel.

\subsubsection{Compressive sensing}

Compressive sensing can be applied as an alternative to reduce the sensing and feedback overhead. In [62], each SU senses linear combinations of multiple narrow bands by selective filters. The results are reported to the SBS, where matrix completion and joint sparsity recovery algorithms are applied to decode the occupied channels. Both algorithms allow exact recovery from incomplete reports and reduce feedback from the SUs to the SBS. Compressive sensing can also be used with other techniques [63].

\subsection{Distributed CSS}

In the centralized CSS, the cooperative SUs need to feed back information to the SBS, which may incur high communication overhead and make the whole network vulnerable to node failure. To address these problems, distributed CSS can be applied.

In the CR networks, an SU can act as a relay for others to improve sensing performance [64-66]. For the scheme in [64], one SU works as a amplify-and-forward (AF) relay for another $\mathrm{SU}$ to get the agility gain when the relay user detects the high PU signal power and the link between two SUs is good. The scheme is extended into multi-user networks [65]. To ensure asymptotic agility gain with probability one, a pairing protocol is developed. Besides AF relay scheme, a detect-and-relay (DR) scheme has been proposed [66], where only the relay SUs that detect the present of the PU signals forward the received signals to the SU transmitter. The results show that DR mode outperforms AF mode.

By using both temporal redundant information in two adjacent sensing periods and the spatial redundant information between two adjacent SUs, a space-time Bayesian compressive CSS for wideband networks has been developed to combat noise [67]. For the multi-hop 
CR networks, a scheme [68] has been proposed to compress the signal in the time domain rather than the power spectral density (PSD) domain by letting each SU estimate PU transmitter and its own signal iteratively, and exchanging information with its neighboring SUs to get the global decision about the availability of the spectrum.

\subsection{Location awareness}

CR networks may be equipped location and environmental awareness features [69] to further improve the performance. A conceptual framework for the locationawareness engine has been developed in [70]. Then, a $\mathrm{CR}$ positioning system has been introduced in [71] to facilitate cognitive location sensing. The location information of PUs and SUs can be used for determining spatial SHs [72]. Moreover, it is very important in public safety CR systems to detect and locate victims [73]. The above is only initial research in the area and more study is desired in the future.

\subsection{Challenges}

\subsubsection{Common control channel}

Common control channel between the SUs and the SBS is assumed in most of existing work, which requires extra channel resources and introduces additional complexity. Moreover, in the CR networks, it is difficult to establish a control channel at the beginning of the sensing stage and the change of the PUs' activities may affect the established control channel. In [74], a selective-relay based CSS scheme without common reporting control channels has been proposed. To limit interference to the PUs, only the relays (SUs) that detect the absence of the PU signal feedback to the SBS. The SBS then uses the received signals that experience fading to make a decision. Compared with the traditional scheme with common reporting control, the proposed scheme does not sacrifice the performance of the receiver operating characteristics (ROC). How to set up and maintain common control channel is still a challenge and an open issue for CR networks.

\subsubsection{Synchronization}

Most study is based on synchronous local observations. However, SUs locate at different places in practical CR systems, resulting in a synchronization problem for data fusion. To enable combination of both synchronous and asynchronous sensing information from different SUs, a probability-based combination method has been proposed in [75] by taking the time offsets among local sensing observations into account.

\subsubsection{Non-ideal information}

Most of the study analyzes the performance of CSS based on the perfect knowledge of the average received SNR of the PU transmitter signal. However, in practice, this is not always the case. The effect of average SNR estimation errors on the performance of CSS has been examined in [76]. In the noiseless-sample-based case, the probability of false alarm decreases as the average SNR estimation error decreases for both independent and correlated shadowings. In the noise-sample-based case, there exists a surprising threshold for the noise level. Below the threshold, the probability of false alarm increases as the noise level increases, where the probability decreases as the noise increases above the threshold.

\section{Spectrum allocation and sharing}

In the previous sections, we have discussed the spectrum sensing techniques for CR networks. Based on the sensing results, the SUs have information about the channels that they can access. However, the channel conditions may change rapidly and the behavior of the PUs might change as well. In order to achieve better system performance, SUs should decide which channel can be used for transmission together with when and how to access the channel. To protect the PU system, the interference generated by the SUs should also be taken into account. Moreover, one SU needs to consider the behavior of other co-existing SUs. In the section, we will discuss the spectrum allocation and sharing schemes to address these problems.

Depending on spectrum bands that the SUs use, the schemes can be divided into two types, namely open spectrum sharing and licensed spectrum sharing $[6,13]$. In the open spectrum sharing system, all the users have the equal right to access the channels. The spectrum sharing among SUs for the unlicensed bands belongs to this type. The licensed spectrum sharing can also be called hierarchical spectrum access model. In such systems, the licensed PUs have higher priorities than the unlicensed SUs. Usually, there are no conflicts among PUs since they all have their own licensed bands. For the SUs, they need to adjust their parameters, such as transmit power and transmission strategy, to avoid the interruption to the PUs. According to the access strategies of the SUs, the hierarchical spectrum access model can be further divided into spectrum underlay and spectrum overlay [13]. In the spectrum underlay system, the SUs are allowed to transmit while the PUs are transmitting. The interference generated from the SUs need to be constrained to protect the PUs. The power control problem is one of the key issues in the systems. In the spectrum overlay systems, the SUs can only transmit when PUs are not or the SUs create interference-free transmission to the PUs by using some advanced techniques. Spectrum overlay is also called opportunistic spectrum access (OSA). 
Another classification depends on whether there exists a central node to manage spectrum allocation and access procedure [6]. The whole procedure may be controlled by a central node. Due to the cost of the central node and information feedback, the centralized approaches may be impractical in some cases. In this case, the SUs may make their own decisions based on the observations of the local spectrum dynamics. This is called distributed spectrum sharing. Of course, several SUs in a system may cooperate with each other, which is called cooperative spectrum sharing [6].

In the following, we will discuss some important techniques on spectrum allocation and sharing.

\subsection{Resource allocation and power control}

In order to limit interference to the PUs created by the SUs, various resource allocation and power control schemes have been proposed for the CR networks.

\subsubsection{Single-carrier and single-antenna systems}

For a point-to-point system with single antenna, the spectrum sharing model can be shown as in Figure 4, where the SU transmitter can transmit as long as interference caused to the PU receiver is below a threshold. The channel gains from the SU transmitter to the SU receiver and the PU receiver are denoted $g_{1}$ and $g_{0}$, respectively. We denote the instantaneous transmit power at the SU transmitter as $P\left(g_{0}, g_{1}\right)$. In such a system, the most common constraints to protect the PUs are peak or average interference powers constraints (IPCs). Under peak IPCs, the overall instantaneous interference power generated by the SUs must be below a threshold, $Q_{p k}$, that is

$$
g_{0} P\left(g_{0}, g_{1}\right) \leq Q_{p k}, \quad \forall\left(g_{0}, g_{1}\right)
$$

Similarly, the constraint on the average interference power can be expressed as

$$
E\left[g_{0} P\left(g_{0}, g_{1}\right)\right] \leq Q_{a v}
$$

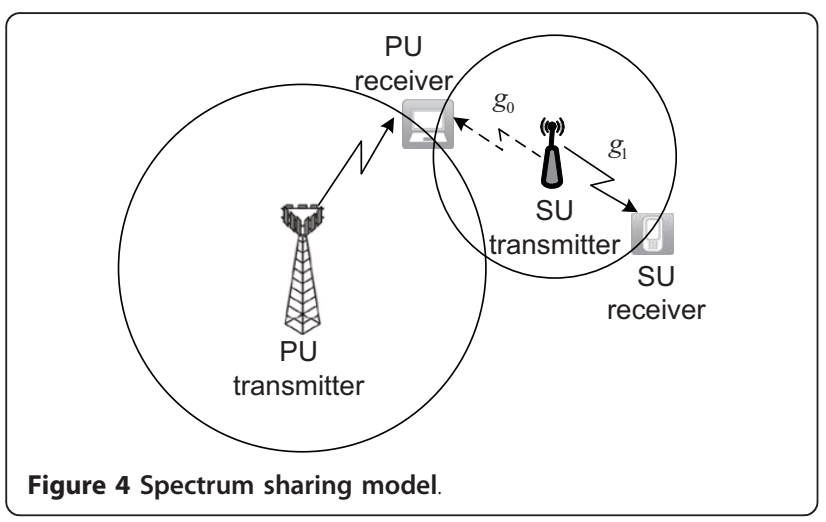

where $Q_{a v}$ is a threshold. Moreover, the transmit power constraints (TPCs) of the SUs should be taken into account. The peak TPC can be expressed as

$$
P\left(g_{0}, g_{1}\right) \leq P_{p k}
$$

where $P_{p k}$ is the peak transmit power limit. The average TPC can be expressed as

$$
E\left[P\left(g_{0}, g_{1}\right)\right] \leq P_{a v}
$$

where $P_{a v}$ is the average transmit power limit.

The power control for systems with single PU pair and single SU pair have been investigated in [77-80]. In [77], different kinds of capacity for the SU system, such as the ergodic, outage, and minimum-rate, are determined for Rayleigh fading environments under both peak and average IPCs. The analysis has been extended to the case with TPCs in [78]. It is shown that the average IPCs can provide higher capacities than the peak average IPCs. If the statistics of any sensing metric conditioned on the PU being ON/OFF are known a priori to the SU transmitter, optimal power control schemes [79] and adaptive rate and power control schemes [80] have been proposed to maximize SU system capacity subject to average IPCs and peak TPCs. The system throughput can be improved using soft information.

More general models with multiple PUs and SUs have been studied in [81]. The power allocation problems for sum-rate maximization on Gaussian cognitive MAC under mutual interferences between the PU and the SU communications are formulated as a standard non-convex quadratically constrained quadratic problem (QCQP) where semidefinite relaxation (SDR) has been applied to find a simple solution.

All the above study focuses on performance analysis for SU systems while the performance of PU systems under average and peak IPCs has been studied in [82]. It has been shown that the average IPCs can be advantageous over the peak IPCs in most cases. Moreover, the existing results demonstrated that the SU system can get better performance under average IPCs. Thus, average IPCs should be used in practice to protect both PU and SU systems.

Besides using IPCs, PU outage probability constraint (OPC) can be used to protect PU transmission [83,84], where the outage probability of the PU transmission should not be below a given threshold. Under the OPC and average/peak TPCs, optimal power allocation strategies have been developed to maximize the ergodic and outage capacities of SU systems in [83]. It has better performance than IPCs. By utilizing the outage information from the PU receiver on the PU feedback channel as an inference signal for coordination, a discounted distributed power control algorithm has been proposed in 
[84] to maximize the utilities of the SUs under OPC and peak TPCs.

\subsubsection{Multi-carrier and multi-channel systems}

In a multi-carrier or multi-channel system, interference generated by the SU to the PU can be considered either in the whole bands/channels or each sub-band (subchannel) separately. Similar to the case of single-carrier and single-antenna systems, the IPCs for the PUs can be divided into two types: peak and average IPCs.

Power control schemes under different constraints for both PU and SU systems have been extensively studied. In [85], capacity maximization for the SU system under TPCs as well as either peak or average IPCs is investigated. It is shown that the average IPC provides better performance for the SU system than the peak IPC. Instead of using IPC directly, optimal power allocation under OPC has been investigated [86]. With the CSIs of the PU link, the SU link, and the SU-to-PU link at the $\mathrm{SU}$, a rate loss constraint (RLC) has been proposed, where the rate loss of the PUs due to the SU transmission should be below a threshold. Under RLC, the transmission efficiency of the SU system increases [87]. From practical point of view, a hybrid scheme by using both IPC and RLC is analyzed as well. Since the spectrum sensing results are not reliable, the probabilistic information of channel availability has been used to assist resource allocation in a multi-channel environment [88]. Compared with the conventional hard decision based IPCs, the proposed approach can utilize the spectrum more efficiently while protect the PUs from unacceptable interference. By considering the SINR requirements for the SUs, downlink channel assignment and power control schemes have been studied under the IPCs to the PUs [89] to maximize the number of active SUs.

Besides the above co-existence scenario, another scenario is in the multi-band system where PU and SU are co-located in the same area with side-by-side bands. For this scenario, power allocation schemes have been proposed in [90]. A risk-return model, which includes these two co-existence scenarios together, has been introduced in [91], and takes into account the reliability of the available sub-bands, their power constraints, and IPCs to the PUs. Besides the optimal power allocation, three suboptimal schemes, namely, the step-ladder, nulling, and scaling schemes have been developed.

\subsubsection{Multi-antenna systems}

For multi-antenna systems, most study jointly optimizes power allocation and beamforming [92-95]. Under IPCs and peak TPCs, the power allocation and beamforming design for sum-rate maximization and signal-to-interference-plus-noise ratios (SINRs) balancing problems have been studied for SIMO systems in [92]. For SINR balancing, all the SUs can achieve their targeted SINRs fairly. When linear minimum mean-square-error (MMSE) receivers are utilized, multiple constraints can be decoupled into several subproblems with a single constraint. The study of SINR-balancing has been extended into MIMO systems in [93], where a robust beamforming design is developed to limit the interference leakage to PU below a specific threshold with a certain probability. Beamforming for MIMO systems has been proposed to maximize the SINR of SUs under IPCs in [94]. A unified homogeneous quadratically constrained quadratic program is used to solve the optimization problems. In practice, it may be impossible that the SINR requirements of all the SUs are satisfied. For this situation, a joint beamforming and admission control scheme has been proposed to minimize the total transmit power of the SU system under IPCs [95].

\subsubsection{Multi-hop systems}

In a relay-assisted system, interference from all relay nodes to the PU receiver should be considered. In [96], transmit power allocation (TPA) schemes among relays have been studied, where overall transmit power is minimized under IPCs as well as the SINR requirement of the targeting SU receiver. A fully distributed TPA has been proposed and provides an almost optimal solution as centralized solution. To reduce the energy consumption of the fully distributed TPA, a distributed feedback assisted TPA has been proposed by feeding back the estimated real-valued transmit power. Besides power allocation, channel allocation has been studied in [97]. In a three-node CR network, end-to-end transmission is categorized into three modes, namely direct, dual-hop, and relay channels. The optimal end-to-end channel and power allocation scheme can be realized by analyzing optimal power allocation for each mode and choosing the mode that can provide the highest system throughput. Joint rate, power control, and channel allocation problem to maximize the throughput of the relayassisted SU system has been studied in [98], where an optimal solution can be obtained by dividing the original problem into a base station (BS) master problem and the relay station subproblems.

\subsection{Spectrum sharing game}

Game theory is a well-developed mathematical tool to model strategic situations, where any individual's success in making choices depends on the choices of others. In the CR networks, the SUs compete for spectrum resources to maximize their own utilities where game theory models can be used [99]. Moreover, the game theory can be also utilized to analyze both the PU and SU systems' behaviors, where they try to maximize their own profits.

From the game-theoretical perspective, power control can be formulated as a distributed game among all the SUs [100], where SUs maximize their own utilities by 
adjusting transmission powers. The total CR network capacity is maximized when the game reaches a Nash equilibrium (NE). In a uplink multiuser CR system, a non-cooperative power control game among SUs can be applied to maximize energy efficiency with a fairness constraint [101]. To jointly design waveform and resource allocation, a general framework has been developed [102], which can be applied to many radio platforms, such as frequency, time, or code division multiplexing, and even agile framework with different expansion functions.

The above study considers SU system only. To take the activities of PUs into account, a dynamic spectrum leasing paradigm based on a game-theoretical framework has been proposed $[103,104]$, which provides an incentive for the PUs to actively allow SUs' transmission.

Besides for the resource allocation, game theory can also have other applications in CR networks, such as stimulating the cooperation between SUs [105] and combating PU emulation attacks $[106,107]$.

However, there are still unaddressed issues in this area. Only spectrum sharing constraints are imposed on the games in most of existing study. Deviations from the ideal games due to non-cooperating SUs or channel constraints still need further study.

\subsection{Spectrum decision}

If there are multiple available bands, the CR networks should be able to decide the best one for each SU. This procedure is called spectrum decision $[108,109]$. Compared to traditional spectrum sharing that is designed as a short-term operation to adapt to the fast time-varying channels, spectrum decision considers long-term channel characteristics. Generally, spectrum decision consists of two main steps: channel characterizing and spectrum assigning. Channel characteristics such as path loss, link errors, and link delay are first obtained based on both current observations of SUs and statistics information of PUs. The best spectrum band is then assigned to each SU. In [109], a spectrum decision framework for CR networks has been discussed. A minimum variancebased and a maximum capacity-based spectrum decision schemes have been developed for real-time applications and best-effort applications, respectively. Moreover, learning mechanisms can be equipped to assist the decision making procedure [110-112]. In [110], the use of reinforcement learning algorithm for spectrum assignment in WCDMA systems has been evaluated to show the satisfactory results under different load conditions. Based on the learning mechanisms, the spectrum decision making problem can also be modeled as a multiarmed bandit problem, which can be solved by upper confidence bound algorithm $[111,112]$. Even though there exists some study about spectrum decision, it is still an open topic.

\subsection{Spectrum handoff}

During the transmission of SUs, the PUs may appear to claim their assigned channels. When this occurs, SUs need to stop transmission, vacate the channels in contention, and find other available channels to continue transmission, which is called spectrum handoff [6]. When a SU changes its spectrum, its transmission suspends, which invariably leads to latency increase. To ensure smooth and fast transition with minimum performance degradation, a good spectrum handoff mechanism is required [113-117]. One way to alleviate latency is to reserve a certain number of spectrum bands for spectrum handoff [113]. SUs immediately use the reserved spectrum bands when it is necessary. However, the number of the reserved bands should be chosen carefully to balance the spectrum efficiency and handoff performance. Another way is proactive spectrum handoff [114], where SUs vacate channels before PUs utilize them to avoid collision. This is obviously too idealistic in many settings unless the $\mathrm{SU}$ is capable of predicting PU behavior as in opportunistic spectrum access. Its performance gain over the reactive spectrum handoff has been demonstrated. To evaluate the latency performance of spectrum handoff, an analytical framework has been proposed in [115]. The effects of general service time distribution, various operating channels, and queueing behaviors of multiple SU connections are also considered.

To achieve reliable continuous transmission, SUs can choose spectrum bands owned by different PUs $[116,117]$. Even if a PU reappears, SUs can continue transmission on others. The spectrum diversity also helps to increase the transmission reliability.

\subsection{Transmission design for CR systems}

In CR networks, the transmission protocols of the SUs can be designed to achieve satisfactory system performance together with fulfilling the IPCs for the PUs.

Relays are the most common choices for increasing SU system performance, as shown in Figure 5. Unlike the traditional cooperation transmission system, the performance of the cooperative SU system decreases by introducing the IPCs. For example, the outage probability may increase [118]. With the increase of the number of relays, the outage performance gap decreases. The relays in the CR networks can assist the transmission between the SU transmitter and receiver to reduce the required transmit power and decrease the interference generated to the PU system [119]. The transmission opportunities created through this way are called as spatial SHs. The relay selection for outage performance 


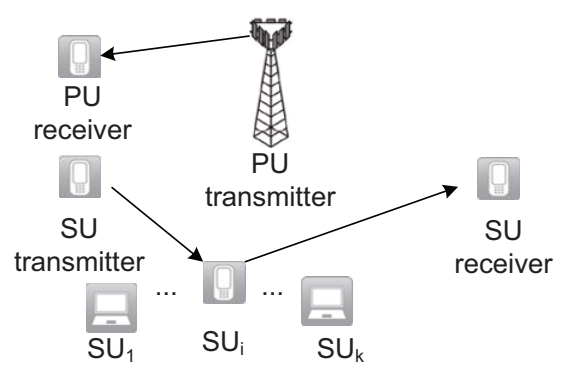

Figure 5 Relay-aided transmission in SU system.

optimization of the SU system under either IPCs [120] or OPC [121] for the PU system have been studied. Power allocation and relay selection can be designed jointly to maximize SU system throughput [122]. Moreover, spectrum sensing and relay selection can be designed jointly $[74,123]$. Without a dedicated relay channel, the best relay is selected for transmission based on the sensing information from the relays that detect the PU signals.

The SU itself can work as a relay to assist PU transmission while transmitting its own data at the same time $[124,125]$. A two-phase transmission protocol in a single antenna system has been considered in [124], where the PU transmits in the first phase and the SU transmitter assigns fractions of the available power to the PU data and its own data for the second phase transmission, respectively. This protocol guarantees the performance of the PU system. With the help of a multi-antenna SU transmitter, both PU and SU systems can achieve better performance by beamforming at the AF SU transmitter [125].

To reduce interference in a MIMO system, the spatial freedom created by antennas can be used. An opportunistic spatial orthogonalization (OSO) scheme [126] has been proposed. With OSO, the SU system can get significant performance improvement without sacrificing of PU system's performance much. Taking the time dimension into account, the interference-free co-existing PU and $\mathrm{SU}$ transmission can be achieved based on orthogonal space-time coding [15].

\subsection{Challenges}

\subsubsection{Joint sensing and access}

In most existing work, the sensing and access techniques are designed separately. However, these two parts are interactive. With different objectives of SU systems, different protection levels for PU systems are needed. It is beneficial to consider these two parts jointly [127]. A joint sensing time and power allocation scheme has been studied in [127] under the average IPCs and TPCs to maximize the ergodic throughput of SU systems. More study is desired in this topic.

\subsubsection{Channel estimation}

The link condition between PU and SU is an important parameter for most of techniques in CR networks. However, the SU-to-PU channel gain is difficult to be obtained by the $\mathrm{SU}$ in practice. To estimate the link condition, the SU can observe PU's behavior by sending out a disturbing probing signal [128] or eavesdrop PU's automatic repeat request feedback information [129]. To avoid estimation of the actual channels between SU transmitter and PUs, a novel practical cognitive beamforming [130] has been proposed based on the effective interference channel, which combines information of the actual channels and the beamforming vectors used by the PUs.

\section{Applications of $\mathbf{C R}$}

The development of spectrum sensing and spectrum sharing techniques enable the applications of CR in many areas. In this section, we introduce some of them.

\subsection{TV white spaces}

The main regulatory agencies for the unlicensed use of TV white spaces are the FCC in the United States, the Office of Communications (Ofcom) in the United Kingdom, and the Electronic Communications Committee (ECC) of the conference of European Post and Telecommunications in Europe. After many years of effort in this area, FCC released the final rules for using the TV white space in September 2010 [131], which led to the culmination of this field. Meanwhile, other agencies have also been getting progress [132]. This is based on the idea of having an accessible database (centralize-fashion) of free TV bands, otherwise called TV white space, or to sense and obtain SHs (distributed-fashion) within TV bands to utilize for SUs communication.

\subsection{Cellular networks}

The applications of CR in cellular networks are emerging in recent years. To overcome the indoor coverage problem and adapt to traffic growth, the concept of small cells, such as femtocells, has been proposed in 3GPP LTE-Advanced (LTE-A) [133] and IEEE $802.16 \mathrm{~m}$ [134], and companies like PicoChip driving femtocell revolution. The femtocell unit has the function of the typical BS (eNodeB in LTE). However, the self-deployment property of the femtocells makes the centralized interference management impractical. Figure 6 illustrates the femtocell interference scenario, where distributed spectrum planning schemes are needed. With $\mathrm{CR}$, the femtocells can search and estimate the available spectrum bands in order to maintain the coverage and avoid the interference to other femtocells and macrocells. 


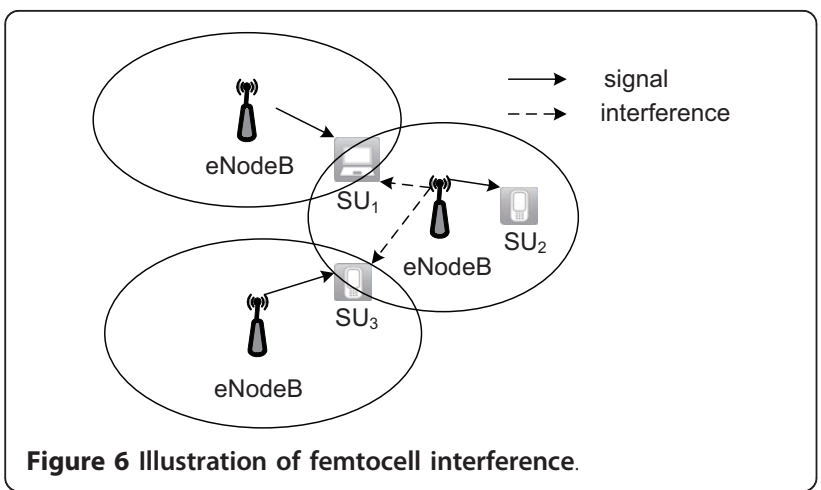

\subsection{Military usage}

$\mathrm{CR}$ is a must-have technique for military usage. With $\mathrm{CR}$, the users can recognize the enemies' communications and protect their own's. Moreover, the users can search for more transmission opportunities. The US department of defense (DoD) has already established programs such as SPEAKeasy radio system and next Generation (XG) to exploit the benefits of CR techniques [6].

\subsection{Emergency networks}

Under severe conditions or extreme situations, e.g., natural disasters and accidents, first re-sponders need to detect and locate survivors and maintain reliable communications between responders and public safety agencies. The infrastructure of the current wireless communication systems is inadequate to meet the future demands of emergency response. The opportunistic spectrum usage provided by $\mathrm{CR}$ techniques could be used to realize efficient and reliable emergency network transmission. The FCC has designated a $700 \mathrm{MHz}$ (698$806 \mathrm{MHz}$ ) frequency band for emergency use [135,136]. The public safety broadband licensee will have priority to access these portions of the commercial spectrum during emergency. Still, there remain some challenges for emergency networks. Possible applications for CR include accurately locating survivors in extreme environments and improving reliability and efficiency of communications. Energy efficiency is particularly important because battery life limits successful operations.

\section{Conclusions}

CR technology has been studied to increase the spectrum utilization efficiency. With spectrum sensing techniques, the SUs are able to monitor the activities of the PUs. To address the limitations of the spectrum sensing techniques by a single SU, CSS schemes have been discussed. Based on the spectrum sensing results, the SUs can access the spectrum bands under the interference limit to the PUs. Different spectrum sharing and allocation schemes have been considered to increase the spectrum efficiency. Even though many critical issues in CR have been addressed in the past decade, there are still some challenges. Nevertheless, we believe the CR technology will be applied to many real systems in the near future.

Due to the space limitation, we have left out some topics, such as security, policy issues, and CR implementation architecture. The readers can refer to some other publications in recent special issues, e.g., [137-140], which may cover these topics.

\section{Acknowledgements}

This study was supported in part by the research project from Sandia National Laboratories.

\section{Competing interests}

The authors declare that they have no competing interests.

Received: 1 May 2011 Accepted: 31 January 2012

Published: 31 January 2012

\section{References}

1. Spectrum Policy Task Force Report, FCC ET Docket 02-155, (2002)

2. J Mitola, Software radios: survey, critical evaluation and future directions. IEEE Aerosp Electron Syst Mag. 8(4), 25-31 (1993)

3. J Mitola, GQ Maguire, Cognitive radio: making software radios more personal. IEEE Personal Commun. 6(4), 13 (1999)

4. S Haykin, Cognitive radio: Brain-empowered wireless communication. IEEE J Sel Areas Commun. 23, 201-220 (2005)

5. FK Jondral, Software-defined radio-basics and evolution to cognitive radio. EURASIP J Wirel Commun Netw. 2005, 275-283 (2005)

6. IF Akyildiz, W-Y Lee, S Mohanty, Next generation/dynamic spectrum access/ cognitive radio wireless networks: A survey. Comput Netw. 50, 2127-2159 (2006)

7. C Stevenson, G Chouinard, Z-D Lei, W-D Hu, S Shellhammer, W Caldwell, IEEE 802.22: The first cognitive radio wireless regional area network standard. IEEE Commun Mag. 47(1), 130 (2009)

8. A Ghasemi, ES Sousa, Spectrum sensing in cognitive radio networks: Requirements, challenges and design trade-offs. IEEE Commun Mag. 46(4), 32-39 (2008)

9. J Ma, GY Li, BH Juang, Signal processing in cognitive radio. Proc IEEE. 97(5), 805-823 (2010)

10. S Haykin, DJ Thomson, JH Reed, Spectrum sensing for cognitive radio. Proc IEEE. 97(5), 849-877 (2010)

11. A Sahai, N Hoven, R Tandra, Some fundamental limits in cognitive radio, in Proc Allerton Conf Commun Control Comput (September 2004)

12. D Cabric, SM Mishra, RW Brodersen, Implementation issues in spectrum sensing for cognitive radios, in Proc Asilomar Conf Signals, Syst Comput (November 2004)

13. Q Zhao, B Sadler, A survey of dynamic spectrum access. IEEE Signal Process Mag. 24(3), 79-89 (May 2007)

14. T Clancy, Achievable capacity under the interference temperature model, in Proc 26th IEEE Int Conf Comput Commun (INFOCOM) (May 2007)

15. VA Bohara, SH Ting, Y Han, A Pandharipande, Interference-free overlay cognitive radio network based on cooperative space time coding, in Proc 5th Int Conf on Cognitive Radio Oriented Wireless Netw and Commun (June 2010)

16. M Ghozzi, M Dohler, F Marx, J Palicot, Cognitive radio: methods for the detection of free bands. Comptes Rendus Physique. 7(7), 74-81 (2006)

17. A Ghasemi, ES Sousa, Impact of user collaboration on the performance of opportunistic spectrum schemes, in Proc IEEE Veh Technol Conf (September 2006)

18. HLV Trees, Detection, Estimation and Modulation Theory: Part I (John Wiley \& Sons Inc., New York, 2001) 
19. A Taherpour, M Nasiri-Kenari, S Gazor, Multiple antenna spectrum sensing in cognitive radios. IEEE Trans Wirel Commun. 9, 814-823 (2010)

20. Z-D Lei, FPS Chin, Sensing OFDM systems under frequency-selective fading channels. IEEE Trans Veh Technol. 6(4), 1960-1968 (2010)

21. E Axell, EG Larsson, Optimal and sub-optimal spectrum sensing of OFDM signals in known and unknown noise variance. IEEE J Sel Areas Commun. 29, 290-304 (2011)

22. A Wald, Sequential Analysis (John Wiley \& Sons Inc., New York, 1947)

23. Z-W Sun, GJ Bradfor, JN Laneman, Sequence detection algorithms for PHYlayer sensing in dynamic spectrum access networks. IEEE J Sel Topics Signal Process. 5(1), 97-109 (2011)

24. A Sonnenschein, PM Fishman, Radiometric detection of spread-spectrum signals in noise of uncertain power. IEEE J Sel Topics Signal Process. 2(1), 4-17 (2008)

25. R Tandra, A Sahai, SNR walls for signal detection. IEEE J Sel Topics Signal Process. 2(1), 4-17 (2008)

26. W Jouini, Energy detection limits under log-normal approximated noise uncertainty. IEEE Signal Process Lett. 18(7), 423-426 (2011)

27. M Ghozzi, F Marx, M Dohler, J Palicot, Cyclostatilonarilty-based test for detection of vacant frequency bands, in Proc 2nd Int Conf on Cognitive Radio Oriented Wireless Netw and Commun (July 2006)

28. PD Sutton, KE Nolan, LE Doyle, Cyclostationary signature in practial cognitive radio applications. IEEE J Sel Areas Commun. 26, 13-24 (2008)

29. W Gardner, Signal interception: A unifying theoretical framework for feature detection. IEEE Trans Commun. 8, 897-906 (1988)

30. Z Quan, WY Zhang, SJ Shellhammer, AH Sayed, Optimal spectral feature detection for spectrum sensing at very low SNR. IEEE Trans Commun. 51, 201-212 (2011)

31. Z Khalaf, A Nafkha, J Palicot, M Ghozzi, Hybrid spectrum sensing architecutre for cognitive radio equipment, in Proc 6th Advanced Int Conf on Telecommun (May 2010)

32. YH Zeng, YC Liang, Eigenvalue-based spectrum sensing algorithms for cognitive radio. IEEE Trans Commun. 57, 1784-1793 (2009)

33. A Kortun, T Ratnarajah, CM Sellathurai, Zhong, On the performance of eigenvalue-based cooperative spectrum sensing for cognitive radio. IEEE J Sel Topics Signal Process. 5(1), 49-55 (2011)

34. T Cui, J Tang, F Gao, C Tellambura, Moment-based parameter estimation and blind spectrum sensing for quadrature amplitude modulation. IEEE Trans Commun. 59, 613-623 (2011)

35. C Cordeiro, M Ghosh, D Cavalcanti, K Challapali, Spectrum sensing for dynamic spectrum access of TV bands, in Proc 2nd Int Conf on Cognitive Radio Oriented Wireless Netw and Commun (July 2007)

36. YC Liang, YH Zeng, ECY Peh, AT Hoang, Sensing-throughput tradeoff for cognitive radio networks. IEEE Trans Wireless Commun. 7, 1326-1337 (2008)

37. Y-L Zou, Y-D Yao, BY Zheng, Outage probability analysis of cognitive transmissions: Impact of spectrum sensing overhead. IEEE Trans Wirel Commun. 9, 2676-2688 (2010)

38. AT Hoang, YC Liang, YH Zeng, Adaptive joint scheduling of spectrum sensing and data transmission in cognitive radio networks. IEEE Trans Commun. 58, 235-246 (2010)

39. H Kim, KG Shin, Efficient discovery of spectrum opportunities with maclayer sensing in cognitive radio networks. IEEE Trans Mobile Comput. 7(5), 533-545 (2008)

40. SH Song, K Hamdi, KB Letaief, Spectrum sensing with active cognitive systems. IEEE Trans Wirel Commun. 9, 1849-1854 (2010)

41. A Sahai, D Cabric, A tutorial on spectrum sensing: Fundamental limits and practical challenges, in Proc IEEE Int Symp on New Frontier in Dynamic Spectrum Access Networks (DySPAN) (November 2005)

42. PP Hoseini, NC Beaulieu, An optimal algorithm for wideband spectrum sensing in cognitive radio systems, in Proc IEEE Int Conf Commun (ICC) (May 2010)

43. P Paysarvi-Hoseini, NC Beaulieu, Optimal wideband spectrum sensing framework for cognitive radio systems. IEEE Trans Signal Process. 59, 1170-1182 (2011)

44. Z Quan, S Cui, AH Sayed, HV Poor, Optimal multiband joint detection for spectrum sensing in cognitive radio networks. IEEE Trans Signal Process. 57, 1128-1140 (2009)

45. D-M Qu, J Ding, T Jiang, X-J Sun, Detection of non-contiguous OFDM symbols for cognitive radio systems without out-of-band spectrum synchronization. IEEE Trans Wirel Commun. 10, 693-701 (2011)
46. J Ma, GD Zhao, YG Li, Soft combination and detection for cooperative spectrum sensing in cognitive radio networks. IEEE Trans Wirel Commun. 7, 4502-4506 (2008)

47. Z Quan, S Cui, AH Sayed, Optimal linear cooperation for spectrum sensing in cognitive radio networks. IEEE J Sel Topics Signal Process. 2(1), 28-40 (2008)

48. G Taricco, Optimization of linear cooperative spectrum sensing for cognitive radio networks. IEEE J Sel Topics Signal Process. 5(1), 77-86 (2011)

49. R Fan, $\mathrm{H}$ Jiang, Optimal multi-channel cooperative sensing in cognitive radio networks. IEEE Trans Wirel Commun. 9, 1128-1138 (2010)

50. M Sanna, M Murroni, Optimization of non-convex multiband cooperative sensing with genetic algorithms. IEEE J Sel Topics Signal Process. 5(1), 87-96 (2011)

51. ECY Peh, YC Liang, Optimization for cooperative sensing in cognitive radio networks, in Proc IEEE Wireless Commun and Networking Conf (WCNC) (March 2007)

52. ECY Peh, YC Liang, YL Guan, YH Zeng, Optimization of cooperative sensing in cognitive radio networks: a sensing-throughput tradeoff view. IEEE Trans Veh Technol. 58, 5294-5299 (2010)

53. W Han, J Li, Z Tian, Y Zhang, Efficient cooperative spectrum sensing with minimum overhead in cognitive radio. IEEE Trans Wirel Commun. 9(3), 3006-3010 (2010)

54. ECY Peh, YC Liang, YL Guan, YH Zeng, Cooperative spectrum sensing in cognitive radio networks with weighted decision fusion schemes. IEEE Trans Wire Commun. 9, 3838-3847 (2010)

55. J Unnikrishnan, $\mathbf{W}$ Veeravalli, Cooperative sensing for primary detection in cognitive radio. IEEE J Sel Topics Signal Process. 2(1), 18-27 (2008)

56. AW Min, X Zhang, KG Shin, Detection of small-scale primary users in cognitive radio networks. IEEE J Sel Areas Commun. 29, 349-361 (2011)

57. C Song, Q Zhang, Cooperative spectrum sensing with multi-channel coordination in cognitive radio networks, in Proc IEEE Int Conf Commun (ICC) (May 2010)

58. P Kaligineedi, VK Bhargava, Sensor allocation and quantization schemes for multi-band cognitive radio cooperative sensing system. IEEE Trans Wirel Commun. 10, 284-293 (2011)

59. A Sahai, R Tandra, S Mishra, N Hoven, Fundamental design tradeoffs in cognitive radio systems, in Proc 1st Int Workshop Technol Policy for Accessing Spectrum (August 2006)

60. Q-Y Zou, SF Zheng, AH Sayed, Cooperative sensing via sequential detection. IEEE Trans Signal Process. 58, 6266-6283 (2010)

61. S-J Kim, GB Giannakis, Sequential and cooperative sensing for multi-channel cognitive radios. IEEE Trans Signal Process. 58, 4239-4253 (2010)

62. J Meng, J Yin, W Li, H Hossain, EH Zhu, Collaborative spectrum sensing from sparse observations in cognitive radio networks. IEEE J Sel Areas Commun. 29, 327-337 (2011)

63. S-J Kim, ED Anese, GB Giannakis, Cooperative spectrum sensing for cognitive radios using kriged kalman filtering. IEEE I Sel Topics Signal Process. 5(1), 24-36 (2011)

64. G Ganesan, YG Li, Cooperative spectrum sensing in cognitive radio part I: Two user networks. IEEE Trans Wireless Commun. 6(6), 2204-2213 (2007)

65. G Ganesan, YG Li, Cooperative spectrum sensing in cognitive radio part II: Multiuser networks. IEEE Trans Wirel Commun. 6, 2214-2222 (2007)

66. Q Chen, M Motani, W-CL Wong, A Nallanathan, Cooperative spectrum sensing strategies for cognitive radio mesh networks. IEEE J Sel Topics Signal Process. 5(1), 56-67 (2011)

67. ZH Zhang, H-S Li, DP Yang, C-X Pei, Space-time bayesian compressed spectrum sensing for wideband cognitive radio networks, in Proc IEEE Int Symp on New Frontier in Dynamic Spectrum Access Networks (DySPAN) (April 2010)

68. F Zeng, C Li, Z Tian, Distributed compressive spectrum sensing in cooperative multihop cognitive networks. IEEE J Sel Topics Signal Process. 5(1), 37-48 (2011)

69. H Celebi, I Guvenc, S Gezici, H Arslan, Cognitive-radio systems for spectrum, location, environmental awareness. IEEE Antennas propag Mag. 52(4), 41-61 (Aug 2010)

70. $\quad H$ Celebi, $H$ Arslan, Utilization of location information in cognitive wireless networks. IEEE Commun Mag. 14(4), 6-13 (Aug 2007)

71. H Celebi, H Arslan, Adaptive positioning systems for cognitive radios, in Proc IEEE Int Symp on New Frontier in Dynamic Spectrum Access Networks (DySPAN) (April 2007) 
72. L Wang, A Chen, Effects of location awareness on concurrent transmissions for cognitive ad hoc networks overlaying infratructure-based systems. IEEE Trans Mobile Comput. 8(5), 577-589 (2009)

73. A Gorcin, Rss-based location awareness for public safety cognitive radio, in Proc IEEE Wireless VITAE (June 2009)

74. Y-L Zou, Y-D Yao, BY Zheng, A selective-relay based cooperative spectrum sensing scheme without dedicated reporting channels in cognitive radio networks. IEEE Trans Wirel Commun. 10(4), 1188-1198 (2011)

75. XW Zhou, J Ma, Li YG, Y Kwon, A Soong, Probability-based combination for cooperative spectrum sensing. IEEE Trans Commun. 58, 463-466 (2010)

76. YF Chen, NC Beaulieu, Performance of collaborative spectrum sensing for cognitive radio in the presence of gaussian channel estimation errors. IEEE Trans Commun. 57, 1944-1947 (2009)

77. L Musavian, S Aissa, Capacity and power allocation for spectrum-sharing communications in fading channels. IEEE Trans Wirel Commun. 8, 148-156 (2009)

78. X Kang, YC Liang, A Nallanathan, HK Garg, R Zhang, Optimal power allocation for fading channels in cognitive radio networks: Ergodic capacity and outage capacity. IEEE Trans Wirel Commun. 8, 940-950 (2009)

79. S Srinivasa, SA Jafar, Soft sensing and optimal power control for cognitive radio. IEEE Trans Wirel Commun. 9, 3638-3649 (2010)

80. V Asghari, S Aissa, Adaptive rate and power transmission in spectrumsharing systems. IEEE Trans Wirel Commun. 9, 3272-3280 (2010)

81. S-W Han, H Kim, Y Han, JM Cioffi, Efficient power allocation schemes for nonconvex sum-rate maximization on gaussian cognitive MAC. IEEE Trans Commun. 58, 753-757 (2010)

82. R Zhang, On peak versus average interference power constraints for protecting primary users in cognitive radio networks. IEEE Trans Wirel Commun. 8, 2112-2120 (2009)

83. X Kang, R Zhang, YC Liang, HK Garg, Optimal power allocation strategies for fading cognitive radio channels with primary user outage constraint. IEEE J Sel Areas Commun. 29, 374-383 (2011)

84. S Huang, X Liu, Z Ding, Decentralized cognitive radio control based on inference from primary link control information. IEEE I Sel Areas Commun. 29, 394-406 (2011)

85. K Son, BC Jung, S Chong, DK Sung, Opportunistic underlay transmission in multi-carrier cognitive radio systems, in Proc IEEE Wireless Commun and Networking Conf (WCNC) (April 2009)

86. K Son, BC Jung, S Chong, DK Sung, Power allocation for OFDM-based cognitive radio systems under outage constraints, in Proc IEEE Int Conf Commun (ICC) (May 2010)

87. X Kang, HK Garg, YC Liang, R Zhang, Optimal power allocation for OFDMbased cognitive radio with new primary transmission protection criteria. IEEE Trans Wirel Commun. 9, 2066-2075 (2010)

88. X Zhou, GY Li, D Li, D Wang, ACK Soong, Probabilistic resource allocation for opportunistic spectrum access. IEEE Trans Wireless Commun. 9, 2870-2879 (2010)

89. AT Hoang, YC Liang, Downlink channel assignment and power control for cognitive radio networks. IEEE Trans Wirel Commun. 7, 3106-3117 (2008)

90. G Bansal, MJ Hossain, VK Bhargava, Optimal and suboptimal power allocation schemes for OFDM-based cognitive radio systems. IEEE Trans Wirel Commun. 7, 4710-4718 (2008)

91. Z Hasan, G Bansal, E Hossain, VK Bhargava, Energy-efficient power allocation in OFDM-based cognitive radio systems: a risk-return model. IEEE Trans Wirel Commun. 8, 6078-6088 (2009)

92. L Zhang, YC Liang, Y Xin, Joint beamforming and power allocation for multiple access channels in cognitive radio networks. IEEE J Sel Areas Commun. 26, 38-51 (2008)

93. ZL Xiong, K Cumanan, S Lambotharan, Robust SINR balancing technique for a cognitive radio network using probability based interference constraints, in Proc IEEE Int Symp on New Frontier in Dynamic Spectrum Access Networks (DySPAN) (April 2010)

94. Y-JA Zhang, AM-C So, Optimal spectrum sharing in MIMO cognitive radio networks via semidefinite programming. IEEE J Sel Areas Commun. 29, 362-373 (2011)

95. MH Islam, YC Liang, AT Hoang, Joint power control and beamforming for cognitive radio networks. IEEE Trans Wirel Commun. 7, 2415-2419 (2008)

96. J Mietzner, L Lampe, R Schober, Distributed transmit power allocation for multihop cognitive radio systems. IEEE Trans Wirel Commun. 8, 5187-5201 (2009)
97. GD Zhao, CY Yang, GY Li, D-D Li, A Soong, Power and channel allocation for cooperative relay in cognitive radio networks. IEEE J Sel Topics Signal Process. 15(1), 151-159 (2011)

98. R Wang, VKN Lau, Y Cui, Decentralized fair scheduling in two-hop relayassisted cognitive OFDMA systems. IEEE J Sel Topics Signal Process. 15(1), 171-181 (2011)

99. Z Ji, KJR Liu, Dynamic spectrum sharing: A game theoretical overview. IEEE Commun Mag. 45, 88-94 (2007)

100. Y-E Lin, K-H Liu, HY Hsieh, Design of power control protocols for spectrum sharing in cognitive radio networks: A game-theoretic perspective, in Proc IEEE Int Conf Commun (ICC) (May 2010)

101. S Buzzi, D Saturnino, A game-theoretic approach to energy-efficient power control and receiver design in cognitive CDMA wireless networks. IEEE J Sel Topics Signal Process. 5(1), 137-150 (2011)

102. Z Tian, G Leus, V Lottici, Joint dynamic resource allocation and waveform adaptation for cognitive networks. IEEE J Sel Areas Commun. 29, 443-454 (2011)

103. SK Jayaweera, G Vazquez-Vilar, C Mosquera, Dynamic spectrum leasing: A new paradigm for spectrum sharing in cognitive radio networks. IEEE Trans Veh Technol. 59(5), 2328-2339 (2010)

104. G El-Howayek, SK Jayaweera, Distributed dynamic spectrum leasing (d-dsl) for spectrum sharing over multiple primary channels. IEEE Trans Wirel Commun. 10, 55-60 (2011)

105. Y-L Wu, BB Wang, KIR Liu, TC Clancy, Repeated open spectrum sharing game with cheat-proof strategies. IEEE Trans Wirel Commun. 8, 1922-1933 (2009)

106. H-S Li, Z Han, Dogfight in spectrum combating primary user emulation attacks in cognitive radio systems, part i known channel statistics. IEEE Trans Wirel Commun. 9, 3566-3577 (2010)

107. H-S Li, Z Han, Dogfight in spectrum: Combating primary user emulation attacks in cognitive radio systems-part ii: Unknown channel statistics. IEEE Trans Wirel Commun. 10, 274-283 (2011)

108. IF Akyildiz, W-Y Lee, MC Vuran, S Mohanty, A survey on spectrum management in cognitive radio networks. IEEE Commun Mag. 46(4), 40-48 (2008)

109. W-Y Lee, IF Akyildiz, A spectrum decision framework for cognitive radio networks. IEEE Trans Mobile Comput. 10(2), 161-174 (2011)

110. N Vucevic, J Perez-Romero, O Sallent, R Agusti, Reinforcement learning for dynamic spectrum management in WCDMA. Telfor J. 1(1), 6-9 (2009)

111. W Jouini, D Ernst, C Moy, J Palicot, Upper confidence bound based decision making strategies and dynamic spectrum access, in Proc IEEE Int Conf Commun (ICC) (May 2010)

112. W Jouini, C Moy, J Palicot, Upper confidence bound algorithm for opportunistic spectrum access with sensing errors, in Proc 6th Int Conf on Cognitive Radio Oriented Wireless Netw and Commun (June 2011)

113. $X$ Zhu, L Shen, T Yum, Analysis of cognitive radio spectrum access with optimal channel reservation. IEEE Commun Lett. 11(4), 304-306 (2007)

114. Y Song, JL Xie, Prospect: A proactive spectrum handoff framework for cognitive radio ad hoc networks without common control channel. IEEE Trans Mobile Comput. (to appear, 2011)

115. L Wang, C Wang, C Chang, Modeling and analysis for spectrum handoffs in cognitive radio networks. IEEE Trans Mobile Comput. (to appear, 2011)

116. H Kushwaha, Y Xing, R Chandramouli, H Heffes, Reliable multimedia transmission over cogntiive radio networks using fountain codes. Proc IEEE. 96(1), 155-165 (2008)

117. D Willkomm, J Gross, A Wolisz, Reliable link maintenance in cognitive radio systems, in Proc IEEE Int Symp on New Frontier in Dynamic Spectrum Access Networks (DySPAN) (November 2005)

118. J Lee, H Wang, JG Andrews, D Hong, Outage probability of cognitive relay networks with interference constraints. IEEE Trans Wirel Commun. 10, 390-395 (2011)

119. GD Zhao, J Ma, GY Li, T Wu, Y Kwon, A Soong, CY Yang, Spatial spectrum holes for cognitive radio with relay-assisted directional transmission. IEEE Trans Wirel Commun. 8, 5270-5279 (2009)

120. Y Han, SH Ting, A Pandharipande, Cooperative spectrum sharing protocol with secondary user selection. IEEE Trans Wirel Commun. 9, 2912-2923 (2009)

121. Y-L Zou, J Zhu, BY Zheng, Y-D Yao, An adaptive cooperation diversity scheme with best-relay selection in cognitive radio networks. IEEE Trans Signal Process. 58, 5438-5445 (2010) 
122. L-Y Li, XW Zhou, H-B Xu, GY Li, DD Wang, A Soong, Simplified relay selection and power allocation in cooperative cognitive radio systems. IEEE Trans Wirel Commun. 10, 33-36 (2011)

123. Y-L Zou, Y-D Yao, BY Zheng, Cognitive transmissions with multiple relays in cognitive radio networks. IEEE Trans Wirel Commun. 10, 648-659 (2011)

124. Y Han, A Pandharipande, SH Ting, Cooperative decode-and-forward relaying for secondary spectrum access. IEEE Trans Wirel Commun. 8, 4945-4950 (2009)

125. R Manna, R Louie, Y Li, B Vucetic, Cooperative amplify-and-forward relaying in cognitive radio networks, in Proc 5th Int Conf on Cognitive Radio Oriented Wireless Netw and Commun (June 2010)

126. C Shen, MP Fitz, Opportunistic spatial orthogonalization and its application in fading cognitive radio networks. IEEE J Sel Topics Signal Process. 5(1), 182-188 (2011)

127. S Stotas, A Nallanathan, Optimal sensing time and power allocation in multiband cognitive radio networks. IEEE Trans Commun. 51, 226-235 (2011)

128. R Zhang, On active learning and supervised transmission of spectrum sharing based cognitive radios by exploiting hidden primary radio feedback. IEEE Trans Commun. 58, 2960-2970 (2010)

129. K Eswaran, M Gastpar, K Ramchandran, Cognitive radio through primary control feedback. IEEE J Sel Areas Commun. 29, 384-393 (2011)

130. R Zhang, F-F Gao, YC Liang, Cognitive beamforming made practical: Effective interference channel and learning-throughput tradeoff. IEEE Trans Commun. 58, 706-718 (2010)

131. Unlicensed Operations in the TV Broadcast Bands, Second Memorandum Opinion and Order, FCC 10-174, (2010)

132. J Wang, M Shosh, K Challapali, Emerging cognitive radio applications: a survey. IEEE Commun Mag. 49(3), 74-81 (2011)

133. Technical Specification Group Radio Access Network; Evolved Universal Terrestrial Radio Access; Medium Access Control Protocol Specification, (Release 8), 3GPP Standard TS 36.321, (2010)

134. System Description Document, IEEE Standard 802.16m, (2009)

135. A Gorcin, H Arslan, Public safety and emergency case communications: opportunities from the aspect of cognitive radio, in Proc IEEE Int Symp on New Frontier in Dynamic Spectrum Access Networks (DySPAN) (October 2008)

136. P Pawelczak, RV Prasad, L Xia, I Niemegeers, Cognitive radio emergency networks-requirements and design, in Proc IEEE Int Symp on New Frontier in Dynamic Spectrum Access Networks (DySPAN) (November 2005)

137. FK Jondral, Cognitive radio: A communications engineering view. IEEE Wirel Commun. 14(4), 28-33 (2007)

138. BB Wang, KIR Liu, Advances in cognitive radio networks: a survey. IEEE J Sel Topics Signal Process. 5(1), 5-23 (2011)

139. C-S Sum, H Harada, F Kojima, Z Lan, R Funada, Smart utility networks in TV white space. IEEE Commun Mag. 49(7), 132-139 (2011)

140. J Mitola, A Attar, H Zhang, O Holland, H Harada, H Aghvami, Special issue on achievements and the road ahead: the first decade of cognitive radio. IEEE Trans Veh Technol. 59(4) (2010)

doi:10.1186/1687-1499-2012-28

Cite this article as: Lu et al:: Ten years of research in spectrum sensing and sharing in cognitive radio. EURASIP Journal on Wireless

Communications and Networking 2012 2012:28.

\section{Submit your manuscript to a SpringerOpen ${ }^{\mathcal{O}}$ journal and benefit from:}

- Convenient online submission

- Rigorous peer review

- Immediate publication on acceptance

- Open access: articles freely available online

- High visibility within the field

- Retaining the copyright to your article

Submit your next manuscript at $\gg$ springeropen.com 